\title{
ORGANIZAÇÃO DO CUIDADO ÀS CONDIÇÕES CRÔNICAS NA ATENÇÃO PRIMÁRIA À SAÚDE DE SOBRAL-CE: AVALIAÇÃO DE PROCESSO NA PERSPECTIVA DE GESTORES
}

Marcos Aguiar Ribeiro, Izabelle Mont'Alverne Napoleão Albuquerque, Isabel Cristina Kowal Olm Cunha, Fenando Daniel de Oliveira Mayorga, Francisco Rosemiro Guimarães Ximenes Neto, Nayana Cíntia Silveira.

\section{RESUMO}

Têm se como objetivo identificar, a partir do processo de trabalho de gestores, as estratégias de organização e gestão do cuidado às condições crônicas Hipertensão Arterial Sistêmica e Diabetes Mellius na Atenção Primária à Saúde. Trata-se de um estudo qualitativo descritivo, de um recorte de uma pesquisa avaliativa. A coleta de dados se deu a partir de entrevistas semiestruturadas realizadas com gestores da saúde e fontes documentes, no município de Sobral-CE, no ano de 2017. Os discursos foram analisados por com suporte do software NVivo11. A partir então, identificou-se que no que se refere a atenção as condições crônicas, foram desenvolvidas estratégias de organização e gestão do cuidado, tais como a instituição de Protocolos, a Estratificação de Risco e o desenvolvimento de processos de Educação Permanente dos trabalhadores. Enfatiza-se a necessidade da modificação de uma atenção prescritiva e centrada na doença para a colaborativa e com foco na pessoa. Em que o cuidado é realizado com base no modo cooperativo, conforme as necessidades dos usuários, com o compartilhamento de responsabilidades, o apoio a autonomia e a decisão compartilhada

Palavras-chave: Avaliação em Saúde; Atenção à Saúde; Doença Crônica.

Revista da Rede APS 2019

Publicada em:

$22 / 03 / 2019$

Marcos Aguiar Ribeiro, (UNIFESP),

Izabelle Mont'Alverne Napoleão Albuquerque, (Universidade Estadual Vale do Acaraú),

Isabel Cristina Kowal Olm Cunha (UNIFESP),

Fenando Daniel de Oliveira Mayorga (UFC),

Francisco Rosemiro Guimarães Ximenes Neto (Universidade Estadual Vale do Acaraú),

Nayana Cíntia Silveira (Universidade Estadual Vale do Acaraú)

Correspondência para: Marcos Aguiar Ribeiro, Brasília, Brasil. marcosribeiroce@gmail.c om 


\section{INTRODUÇÃO}

O Brasil vivencia uma transição epidemiológica singular, diferente da existente nos países desenvolvidos, explícita na tríplice carga de doenças, com uma agenda não superada das doenças transmissíveis, uma quantidade importante de causas externas e uma presença hegemônica das Doenças Crônicas não Transmissíveis. Nesta conjuntura, destaca-se o predomínio das condições crônicas, que se apresentam de maneira persistente e demandam respostas sociais dos sistemas de atenção à saúde, dos profissionais de saúde e das pessoas usuárias (MENDES, 2012).

Dentre as condições crônicas, a Hipertensão Arterial Sistêmica (HAS) e a Diabetes Mellitus (DM) se configuram como epidemias mundiais e representam um grande desafio para os sistemas de saúde de forma que afeta a vida de milhões de pessoas e resultam em grande impacto econômico e alto custo social (WHO, 2016; YUSUFALI et al, 2016).

Nesta perspectiva, a atual situação de saúde de predomínio das condições crônicas não pode ser respondida de maneira eficiente, efetiva e de qualidade por sistemas de saúde voltados, prioritariamente, para as condições agudas e para as agudizações de condições crônicas (MENDES, 2011). Assim, faz-se necessário a transição no sistema de saúde, por meio da operacionalização de um modelo com foco na atenção às condições crônicas por meio de mudanças na organização da prestação de serviços, na tomada de decisões, nos sistemas de informação clínica, no autocuidado apoiado e na articulação eficaz com outros serviços de saúde. Para assim, potencializar usuários informados e ativos no seu cuidado, bem como equipe de saúde preparada e proativa para fornecer melhores resultados na saúde (MENDES, 2012).

Diante disso, a Atenção Primária à Saúde (APS) tem papel fundamental nas estratégias de prevenção, diagnóstico, monitorização e controle das condições crônicas, com foco na pessoa de forma integral, devendo envolver os usuários na definição e implementação de estratégias de controle e autocuidado, considerando os determinantes e condicionantes envolvidos. Além disso, a APS deve atuar como coordenadora do cuidado, promovendo uma atenção à saúde de qualidade e auxiliando no manejo das demandas e necessidades de saúde dos usuários, atuando conforme o modelo de atenção centrado nas condições crônicas de saúde (BRASIL,2013).

Assim, têm-se como objetivo identificar, a partir do processo de trabalho de gestores, as estratégias de organização e gestão do cuidado às condições crônicas HAS e DM na Atenção Primária à Saúde.

\section{Metodologia}

Trata-se de uma pesquisa do tipo avaliativa com abordagem qualitativa, onde se utilizou a adaptação de uma matriz de avaliação de efetividade de intervenções para controlar e prevenir Doenças Crônicas Não Transmissíveis empregado na América Latina proposta por De Salazar (2011), e utilizada no Brasil por Moysés; Silveira Filho; Moysés (2012).

Neste recorte, têm-se como enfoque a avaliação do processo de atenção às condições crônicas a partir das narrativas gerentes e tutores do Sistema de Saúde de Sobral, considerados os stakeholders para o entendimento dos processos de gestão do cuidado às condições crônicas HAS e DM.

O estudo foi realizado no município de Sobral, no Ceará, o qual constitui cenário de referência para a Região Nordeste do Brasil no que se refere à singularidade e às inovações do seu modelo técnico-assistencial e cobertura da Estratégia Saúde da Família

A coleta de dados aconteceu em 2017, a partir de entrevistas semiestruturadas realizadas com quatro tutores do sistema de saúde (apoiadores institucionais) e 18 gerentes da Estratégia Saúde da Família. Além disso, foram acessadas fontes documentais para o processo de análise qualitativa. Neste sentido, foram coletados dados a partir de entrevistas com gerentes e tutores do Sistema de Saúde, Protocolo da Hipertensão Arterial Sistêmica, Protocolo da Diabetes mellitus, Cronograma de Atendimento na APS, Instrumento do fluxo do Acolhimento à demanda Espontânea, Parâmetro para a Estratificação de Risco da Hipertensão e Diabetes, Consolidado da Territorialização. 
Os discursos foram analisados a partir de uma análise textual descritiva e discursiva, onde foram descritos os achados e os sentidos possíveis com vistas à ampliação das compreensões relacionadas à atenção às condições crônicas, com suporte do software N Vivo 11.

Ressalta-se que o estudo seguiu os preceitos da Resolução no 466/2012 do Conselho Nacional de Saúde, com parecer favorável do Comitê de Ética sob no 2.054.329 (BRASIL, 2012a).

\section{RESULTADOS E DISCUSSÕES}

A partir da análise dos discursos, foram identificados os núcleos de sentido, a saber: Gestão da Clínica, Autocuidado apoiado, Estratégias de enfrentamento de fatores restritivos da atenção, Experiências exitosas e Educação Permanente em Saúde. Assim, estes núcleos de sentido, configuraram-se como elementos constitutivos de um conjunto que caracterizaram as estratégias de organização e gestão do cuidado às condições crônicas.

Dentre as estratégias de organização da atenção às condições crônicas, destacam-se um conjunto de tecnologias de microgestão da clínica, destinado a promover e qualificar a atenção às condições crônicas, embasado por evidências científicas (MENDES, 2011).

Neste sentido, os protocolos são tidos como instrumentos orientadores para o enfrentamento de diversos problemas na assistência e na gestão dos serviços. São orientados por diretrizes de natureza técnica, organizacional e política e têm como fundamentação estudos validados pelos pressupostos das evidências científicas e que servem para orientar fluxos, condutas e procedimentos clínicos dos trabalhadores dos serviços de saúde (GIL; LUIZ; GIL, 2016).

Diante da necessidade de organização dos serviços de saúde advinda das mudanças na carga de doenças, Sobral instituiu dois protocolos, a saber: Diretriz para Screening, Diagnóstico e Tratamento do Diabetes Mellitus tipo 2 em Adultos e Diretriz para Screening, Diagnóstico e Tratamento da Hipertensão Arterial Sistêmica em Adultos. Esse processo de construção dos protocolos contou com o apoio dos coordenadores, tutores, gestores e os profissionais da atenção Básica:

"protocolo foi construído participativamente com os profissionais da estratégia de saúde da família, médicos, enfermeiros e equipe multiprofissional, NASF (E3)".

Assim, o uso de diretrizes e protocolos pelas equipes de Saúde está fortemente relacionado à melhor qualidade da assistência, resultando em diagnósticos mais precisos, em tratamentos mais adequados, em melhor uso de recursos e exames e em melhores resultados em Saúde. Dessa forma, o uso de protocolos visa à melhoria da assistência e direcionam os cuidados ás ações estabelecidas, otimizam recursos, e proporcionam eficiência e qualidade no cuidado (MENDES, 2012). Neste sentido, os discursos também reverberam os avanços na atenção com a instituição dos protocolos:

"Antes do protoloco era uma bagunça, os profissionais não tinham o Norte sobre o que fazer como fazer. Eram seguidos os manuais do ministério, mais não era toda a rede que seguia, pois não tinha um protocolo adaptado para as nossas peculiaridades culturais e territoriais(E1)."

Portanto, ficam evidentes os avanços obtidos com a implementação dos protocolos, visto que por meio deste, é possível ordenar os fluxos de atendimento, identificando os pacientes com doenças crônicas e estratificando os riscos de cada um, e dessa forma poder direcionar os encaminhamentos de acordo com as necessidades especificas. Além disso, o reconhecimento das singularidades locais nos protocolos possibilita uma coerência entre pensar e agir em saúde.

O desenvolvimento de protocolos facilita a ação da regulação, pois permitem que a gestão estabeleça quais são os usuários que precisam ser avaliados pelo serviço especializado e aqueles que não têm necessidade de atendimento em outro nível de atenção e podem ser manejados na APS (BARBOSA; BARBOSA; NAJBERG, 2016). Esse fato corrobora com as seguintes falas: 
"todas as equipes são cientes desse protocolo, onde nele está contido a questão da estratificação de risco, a periodicidade que cada paciente deve realizar exames, consultas especializadas ou com médico generalista, enfim nós temos esse protocolo, e todas a equipe tem acesso a ele. (E4)".

Todavia, a realidade da rotatividade dos profissionais da ESF dificulta o desenvolvimento das diretrizes definidas pelos protocolos:

"[...] a gente tem uma grande rotatividade de profissionais eu lembro que antes a gente sentou todos enfermeiros, estudamos, e na outra semana não tinha nenhuma enfermeira daqueles que estavam ali. Eram outros! E a gente vai nessa coisa de fazer tudo de novo essa rotatividade é muito grande e aí entrou pessoas que nem participaram da construção desse protocolo que nem conhecia. Isso é uma coisa que dificulta muito (E8)".

Assim, é importante considerar que as condições de trabalho dos profissionais da saúde vêm sendo degradadas desde há muito, por mecanismos de precarização, que vão desde a não efetivação de profissionais em quantidade suficiente até a implantação de programas de expansão inconsequentes (SILVA; OLIVEIRA; PEREIRA, 2015), o que implica na grande rotatividade dos profissionais da ESF.

Outra estratégia que vem sendo implementada é a Estratificação de Risco da HAS e DM no âmbito da APS. A Estratificação de Risco das pessoas com condições crônicas consiste em um processo por meio do qual se identificam os grupos ou estratos de risco relacionados a uma determinada condição de saúde, prioritariamente a condição crônica, considerando a severidade da condição e a capacidade de autocuidado (BRASIL, 2013).

Conhecer a população usuária de um sistema de atenção à saúde é o elemento básico que torna possível romper com a atenção baseada na oferta, característica dos sistemas fragmentados, e instituir a atenção baseada nas necessidades de saúde da população, elemento essencial das Redes de atenção (ARRUDA et al, 2015). Diante disso destaca- se a importância da estratificação de risco nesse processo:

"no protocolo existe a estratificação de risco, risco baixo, moderado e alto [...] organizamos os atendimentos de acordo grau de classificação do paciente, eles possuem retornos mensais ou semanais, se houver necessidade. Se for um paciente que tem uma adesão boa ao tratamento, esses retornos são espaçados para cada três meses ou cinco meses, dependendo do uso da adequado, da adesão ao tratamento, da própria orientação do paciente e se o paciente possui um apoiador desse tratamento. Pacientes de risco elevado retornam à unidade semanalmente, até conseguirmos melhorar a classificação de risco desse paciente para um risco mais leve (E12)."

“[...] a partir da estratificação conseguimos rever cronogramas de atenção flexíveis e que trabalha das diversas maneiras, a pessoa que vai ter o atendimento individual, a pessoa que vai ter atendimento compartilhado, a pessoa que vai se beneficiar mais da atenção especializada (E1)".

Neste sentido, a estratificação de risco direciona as ações no âmbito da APS, com vistas a possibilitar uma atenção singular e coerente. A Figura 1 evidencia as ações predominantes por extrato:

Figura 1. Ações de Saúde Predominantes de acordo com a Estratificação de Risco de usuários com HAS e DM na Atenção Primária à Saúde. Sobral, Ceará, Brasil (2019).

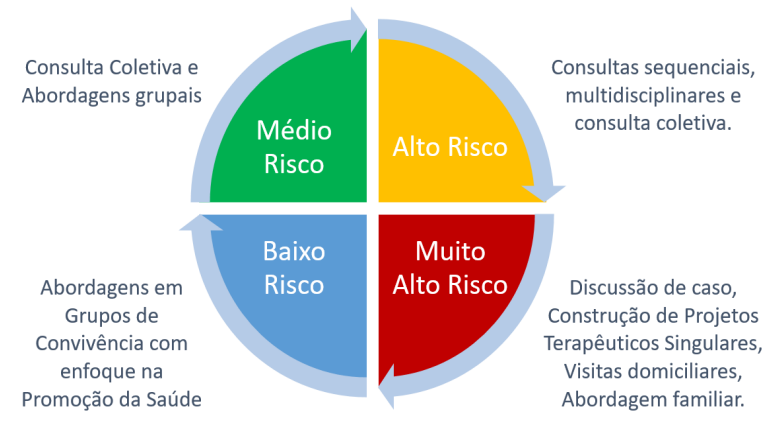

Fonte: Elaborado pelos autores 
Deste modo, as abordagens em grupos de convivência com enfoque na Promoção da Saúde, possibilitam uma atenção por meio de atividades coletivas direcionadas aos fatores de risco. Além disso, o olhar singular para usuários com HAS e DM permitem o desenvolvimento de uma atenção individual/compartilhada em atividade de grupo, com enfoque no Autocuidado apoiado, bem como estratégias de gestão de caso para usuários de muito alto risco, tais como a discussão de caso, a construção de Projetos Terapêuticos Singulares (PTS), Visitas domiciliares (VD) e uma abordagem familiar.

Nesta perspectiva, a partir da estratificação de risco, pode-se garantir que as pessoas com condições crônicas de menores riscos têm sua condição centrada em tecnologias de autocuidado apoiado e com foco na APS, enquanto que os portadores de condições de alto e muito alto risco têm uma presença mais significativa de atenção profissional, com uma concentração maior de cuidados pela equipe de saúde e com a coparticipação da APS e da atenção especializada (MENDES, 2012). Assim, o autocuidado é uma importante estratégia:

“Então um autocuidado apoiado é um componente estratégico na atenção às pessoas com condições crônicas. Então, temos que investir muito no autocuidado a partir da incorporação de tecnologias leves (E15)".

O autocuidado apoiado significa uma colaboração estreita entre a equipe de saúde e os usuários, os quais trabalham em conjunto para definir o problema, estabelecer as metas, monitorá-las, instituir os planos de cuidado e resolver os problemas que apareçam ao longo do processo de manejo (MENDES, 2011).

Assim, o sucesso do tratamento depende fortemente da participação e do envolvimento do usuário enquanto sujeito ativo de seu tratamento. Uma atitude de autocuidado que leve a estilos e práticas de vida mais saudáveis, assim como a adesão ao tratamento, não depende apenas de uma prescrição profissional, mas de uma conscientização do usuário sobre sua condição de saúde e a relação dela com suas práticas (MENDES, 2012).

A capacidade do autocuidado refere-se ao grau de confiança e o apoio que as pessoas têm para cuidar de si mesmas. Pode ser avaliada pela percepção do profissional de saúde, com conhecimento das atitudes da pessoa frente à condição crônica e do seu contexto. Desta maneira, relaciona-se aos seguintes aspectos: Conhecimento e crenças sobre a condição de saúde; Atitudes, confiança e motivação frente às mudanças; Importância dada à condição; Presença e força das redes de suporte social e familiar.

Verificam-se ainda, experiências e estratégias que estão sendo desenvolvidas de forma singular em diferentes territórios e que podem ser exemplo para o enfrentamento dos aspectos restritivos e para qualificação da atenção:

"tem uma experiência muito legal que é o acolhimento multiprofissional e o atendimento descentralizado que é realizado semanalmente [...] em espaço cedido pela associação do bairro. No momento acontece atividade educativa, aferição de pressão arterial, verificação de glicemia e peso e consulta de enfermagem. Os atendimentos são para os usuários com diabetes e hipertensão, tendo em vista a dificuldade do acesso ao CSF, devido a distância e fragilidade na locomoção dos mesmos. Com esse atendimento descentralizado é perceptível a satisfação dos usuários e maior qualidade na adesão ao tratamento, diminuindo este público na demanda espontânea do CSF (Consolidado Territorialização)"

Identificam-se ainda experiências de estímulo às Práticas Integrativas e Complementares:

"A gente trabalha com a fitoterapia, a gente tenta reduzir a medicalização dos pacientes na unidade. Então criamos o projeto Farmácia Viva, onde temos uma horta de planta medicinais [...] nós mandamos as plantas pra a universidade onde são produzidos os medicamentos fitoterápicos. A partir dessa produção a gente conseguiu 
reduzir mais de $30 \%$ a ingestão de medicamentos aqui na unidade, no que diz respeito aos medicamentos complementares ao tratamento de hipertensos e diabéticos. Como por exemplo, quando a gente chegou tinha um determinado profissional que prescrevia para todos os pacientes que eram hipertensos e diabéticos um medicamento protetor gástrico, era um consumo de medicamentos diário, ele achava que todos os pacientes deveriam tomar esse medicamento, e mesmo sem sintomas o paciente tomava e quando fomos tentar fazer o desmame tivemos muita resistência popular. Então a gente precisava pensar em uma estratégia e ai com os fitoterápicos, foi produzido um lambedor, feito de uma erva que é um protetor gástrico, agente prescreve, os próprios enfermeiros podem prescrever os medicamentos fitoterápicos, eles foram capacitados para isso, a equipe toda, os enfermeiros e médicos, e eles passam esse fitoterápico quando há necessidade, quando 0 paciente tem sintomatologia (E8)".

O Ministério da Saúde, por meio do Caderno da Atenção Básica: Práticas integrativas e complementares: plantas medicinais e fitoterapia na Atenção Básica, evidencia os benefícios da oferta de ações/serviços de fitoterapia como prática integrativa e complementar na atenção básica de revitalização do conhecimento popular/tradicional das comunidades, por meio da troca de saberes e a construção do conhecimento sobre plantas medicinais, fortalecendo o seu uso racional, favorecendo maior envolvimento do usuário em seu tratamento, estimulando sua autonomia e corresponsabilização. Além disso, são novas opções terapêuticas às situações clínicas apresentadas no cotidiano das ESF o que torna possível a redução da medicalização excessiva (BRASIL, 2012b).

Foram abordadas ainda, estratégias de enfrentamento dos problemas de desabastecimento de medicação:

"A farmacêutica no passado identificou muitos usuários que tinham medicamentos em casa que não tomava mais, muitas vezes por alteração no projeto terapêutico e então fizemos 0 resgate desses medicamentos (E2)".

Assim, o desenvolvimento destas estratégias colabora para que a APS organize o cuidado aos usuários com HAS e DM, de modo que a partir do processo de estruturação de ferramentas de classificação das prioridades, possibilita-se uma atenção resolutiva coerente com as singularidades dos usuários e família, bem como o compartilhamento do cuidado em rede.

Salienta-se ainda, que todo o processo de transformação da organização e gestão do cuidado às condições crônicas foi amparado pelo desenvolvimento de processo de educação permanente dos trabalhadores do SUS:

"[...] foi feito a formação para eles de tecnologias leves e periodicamente vivenciamos processos formativos para subsidiar a qualificação da atenção as pessoas com hipertensão e diabetes (E1)"

A Educação Permanente em Saúde (EPS) é o conceito pedagógico capaz de implementar relações orgânicas entre ensino, serviço, gestão e controle social. Dessa forma, agrega aprendizado, reflexão crítica sobre o trabalho e resolutividade da clínica e da promoção da saúde coletiva. Neste sentido, a EPS é capaz de potencializar o lócus de atuação profissional a partir da conexão entre educação e trabalho, no qual o aprender e o ensinar incorporam-se ao cotidiano das organizações. Assim, baseia-se na aprendizagem significativa, que promove e produz sentidos e desenvolve-se a partir dos problemas diários e do contexto local, considerando os conhecimentos e as experiências preexistentes das equipes de saúde (CECCIM, FEURWERKER, 2004; LEMOS, 2016).

Desse modo, os discursos também evidenciam a importância dos processos de educação permanente que acontecem em espaços não formais, na própria unidade, onde a equipe se implica na organização, facilitação e planejamento da Educação Permanente, de modo a sair da condição de mero receptor de informações, passando a construir o conhecimento significativo para a transformação da práxis. 
Nesse sentido, a EPS representa um esforço voltado a transformar as práticas de trabalho a partir de sua ressignificação, de sua atualização, de sua reconstrução e de sua reorientação (CECCIM, FEURWERKER, 2004, LEMOS 2016).

Verifica-se também as contribuições do projeto o projeto de Qualificação da Atenção Primária a Saúde (Qualifica APSUS) com um processo de Educação Permanente. O Qualifica APSUS é um projeto da Secretaria do Estado do Ceará com o objetivo de apoiar os municípios no fortalecimento da APS, principalmente quanto a reorganização do modelo de atenção nos municípios e a implementação das Redes de Atenção (RAS), a fim de estabelecer uma linguagem comum nos diferentes pontos de atenção à saúde (CEARÁ, 2017):

“[...] a gente está com a oficina da qualificaAPSUS, que essa oficina ela nos atenta a isso, a ter um atendimento mais voltado as doenças crônicas, ao cuidado continuado (E13)".

Esse projeto a qualificação do cuidado às condições crônicas busca fortalecer o cuidado integral aos usuários, para obter melhores resultados sanitários e melhor satisfação pelos usuários, garantindo uma atenção primária resolutiva que atenda de forma integral as necessidades dos usuários do SUS.

\section{CONSIDERAÇÕES FINAIS}

$\mathrm{Na}$ atenção às condições crônicas hipertensão e diabetes na APS identifica-se um interesse e investimento da gestão da atenção em implementar um modelo de atenção que atenda com integralidade às condições crônicas, desse modo, foram desenvolvidas estratégias de organização e gestão do cuidado, tais como a instituição de Protocolos com diretrizes para Screening, Diagnóstico e Tratamento da HAS e DM, a Estratificação de Risco da HAS e DM e o desenvolvimento de processos de Educação Permanente dos trabalhadores do sistema, disparados pela gestão municipal e estadual e principalmente pelos profissionais da APS no cotidiano do trabalho.
Diante disso, enfatiza-se a necessidade de uma transição de um modelo de agudização da doença para implantar o modelo de atenção às condições crônicas, assim como a modificação de uma atenção prescritiva e centrada na doença para a colaborativa e com foco na pessoa. Em que o cuidado é realizado com base no modo cooperativo, conforme as necessidades dos usuários, com a participação da família e empoderamento dos indivíduos, deixando de ser paciente para se tornar produtor e agente de sua saúde. Ainda, ressalta o compartilhamento de responsabilidades, o apoio a autonomia e a decisão compartilhada, que favorece para uma melhor satisfação do usuário e consequentemente, adesão ao tratamento, obtendo melhores resultados.

\section{AgRAdECIMENTOS}

A Fundação Cearense de Apoio ao Desenvolvimento Científico e Tecnológico (Funcap) pelo financiamento do projeto e a Coordenação de Aperfeiçoamento de Pessoal de Nível Superior (CAPES) pela concessão da bolsa de estudos.

\section{REFERÊNCIAS BIBLIOGRÁFICAS}

(1) ARRUDA, $C$ et al. Redes de atenção à saúde sob a luz da teoria da complexidade. Esc. Anna Nery, Rio de Janeiro, v. 19, n. 1, p. 169-173, Mar. 2015. Disponível em: <http://www.scielo.br/scielo.php?script=sci_arttext \&pid=S1414$81452015000100169 \&$ lng=en\&nrm=iso $>$. Acesso em: 10 Jan. 2018.

(2) BARBOSA, D.V.S.; BARBOSA, N.B.; NAJBERG, E. Regulação em Saúde: desafios à governança do SUS. Cad. saúde colet., Rio de Janeiro, v. 24, n. 1, p. 49-54, Mar. 2016. Disponível em: <http://www.scielo.br/scielo.php?script=sci_arttext \&pid=S1414462X2016000100049\&lng=en\&nrm=iso>. Acesso em: 03 Jul. 2018.

(3) BRASIL. Ministério da Saúde. Estratégias para o Cuidado da Pessoa com Doença Crônica: Hipertensão Arterial Sistêmica. Brasília, DF, 2013. 
(4) BRASIL. Conselho Nacional de saúde. Resolução de no 466, de 12 de dezembro de 2012. Dispõe sobre pesquisas envolvendo seres humanos. Brasília, DF, 2012a.

(5) BRASIL. Ministério da Saúde. Práticas integrativas e complementares: plantas medicinais e fitoterapia na Atenção Básica. Brasília, DF, 2012b.

(6) CEARÁ. Secretaria da Saúde. Projeto Qualifica APSUS Ceará: Oficina 1. 2017. Disponível em:

http://www.saude.ce.gov.br/index.php/downloads/ category/140-apostilas\#>. Acesso em 19 out. 2017.

(7) CECCIM, R.B.; FEUERWERKER, L.M. O quadrilátero da formação para a área da saúde: ensino, gestão, atenção e controle social. Physis., v.14, n.1, p.41-65. 2004. Disponível em: < http://www.scielo.br/scielo.php?pid=S0103-

$73312004000100004 \&$ script=sci_abstract \&tIng=pt>. Acesso em: 03 jan. 2018.

(8) DE SALAZAR, L. ¿'Funcionan y son efectivas las intervenciones para prevenir y controlar las enfermedades crónicas? ¿Qué dice la evaluación? Santiago de Cali: Colección Ciencias Sociales, 2011.

(9) GIL, R.R; LUIZ, I.C; GIL, M.C.R. Gestão pública em saúde: a Importância do planejamento. EDUFMA, São Luís, 2016.

(10) LEMOS, C.L.S. Simão. Educação Permanente em Saúde no Brasil: educação ou gerenciamento permanente? Ciênc. saúde coletiva, Rio de Janeiro, v. 21, n. 3, p. 913-922, Mar. 2016. Disponível em: <http://www.scielo.br/scielo.php?script=sci_arttext \&pid=S1413-

$81232016000300913 \& \operatorname{lng}=e n \& n r m=i s o>$. Acesso em: 10 Dez. 2018.
(11) MENDES, E.V. As redes de atenção à saúde. Brasília: OPAS, 2011. 549p.

(12) MENDES, E.V. O cuidado das condições crônicas na atenção primária à saúde: o imperativo da consolidação da estratégia da saúde da família. Brasília: Organização Pan-Americana da Saúde, 2012.

(13) MOYSÉS, S. T.; SILVEIRA FILHO, A. D.; MOYSÉS, S. J. (Org.). A implantação do modelo de atenção as condições crônicas em Curitiba: resultados do laboratório de inovação sobre atenção às condições crônicas na atenção primária em saúde. Brasília: OPAS/CONASS, 2013. 390 p.

(14) SILVA, R.M.C.A.; OLIVEIRA, D.C.; PEREIRA, E.R. A produção discursiva dos profissionais acerca da humanização em saúde: singularidade, direito e ética. Rev. Latino-Am. Enfermagem, v. 23, n.5, p.936-44. 2015. Disponível em: < http://www.scielo.br/pdf/rlae/v23n5/pt_01041169-rlae-23-05-00936.pdf>. Acesso em: 02 jan. 2018

(15) WORLD HEALTH ORGANIZATION (WHO). Diabetes: Nota descritiva [internet]. Novembro, 2016. Disponível em: http://www.who.int/mediacentre/factsheets/fs312 /es/. Acesso em 30 dez 2018.

(16) YUSUFALI, A. et al. Prevalence, awareness, treatment, and control of hypertension in the middle east: results from the prospective urban rural epidemiology (pure) study. Journal of Hypertension, v. 34, p.551, 2016. Disponível em: <http://journals.Iww.com/jhypertension/Fulltext/2 016/09001/LBOS_03_01_PREVALENCE,_AWARENES S,_TREATMENT,_AND.1635.aspx?trendmdshared $=\% 25$ SPONSORED\%25>. Acesso: 30 jan. 2017. 


\section{ABStRact}

The objective of this study is to identify, from the work process of managers, the strategies of organization and management of care to the chronic conditions Systemic Arterial Hypertension and Diabetes Mellius in Primary Health Care. This is a qualitative descriptive study, a cut-off of an evaluative research. Data collection was based on semi-structured interviews conducted with health managers and documentary sources, in the municipality of Sobral-CE, in the year 2017. The discourses were analyzed by the software NVivo11. From that point onwards, it was identified that in terms of chronic conditions attention has been given to strategies of organization and management of care, such as the establishment of Protocols, Risk Stratification and the development of Permanent Education processes for workers. Emphasis is placed on the need to modify prescriptive and disease-centered attention to collaborative and person-centered approaches. In which care is performed based on the cooperative mode, according to the needs of the users, with the sharing of responsibilities, support for autonomy and shared decision.

Keywords: Health Assessment; Health Care; Chronic disease. 